\title{
Significance and Impact of the Intersphincteric Plane Sepsis in the Management of the Perianal Sepsis: Ultrasound Study
}

\author{
Ashraf Talaat Youssef ${ }^{1}$ \\ ${ }^{1}$ Department of Radiology, Faculty of Medicine, Fayoum University, \\ Fayoum, Egypt
}

Address for correspondence Ashraf Talaat Youssef, MD, Department of Radiology, Faculty of Medicine, Fayoum University, Fayoum, Egypt (e-mail: ashraftalaat1@yahoo.com).

\author{
Abstract \\ Keywords \\ - intersphincteric plane \\ sepsis \\ - management of \\ perianal fistula \\ - anorectal abscess \\ - pathogenesis of \\ perianal fistula \\ - transperineal \\ ultrasound
}

Background The intersphincteric plane (ISP) is a potential space between the external and the internal anal sphincters. About $90 \%$ of the perianal sepsis is caused by an obstruction of the ducts of anal glands with subsequent secondary bacterial infection. The imaging modalities used to diagnose perianal sepsis are the ultrasound via endoanal and transperineal routes and magnetic resonance imaging.

Objective The study aimed to identify the various patterns of the ISP sepsis and their incidence among our study group and to clarify the significance of ISP in the pathogenesis of anorectal abscesses and various types of perianal fistula tracts, to optimize the surgical management.

Methods Retrospective descriptive study in which 57 patients with an ISP sepsis were evaluated with full medical history, clinical exam, and ultrasound; the final diagnosis was based on combining the results of ultrasound, digital rectal examination under anesthesia, and the operative results.

Results The current study showed many patterns of the ISP sepsis and estimated the incidence of each pattern among our study group; such patterns could be fistula tracts, sinus tracts, abscesses, intersphincteric abscess with supralevator extension, sinus with abscess formation, fistula with abscess formation, distension of the ISP with free pus, and other complex patterns like horse abscess, abscess with transsphincteric sinus, and ISP fistula/sinus with transsphincteric branches.

Conclusion Understanding the role of the ISP in the pathogenesis of anorectal abscesses and perianal fistula tracts can help in reaching the optimum way of management.

\section{Introduction}

The intersphincteric plane (ISP) is a potential space between the external and the internal anal sphincters. It communicates with the perianal space inferiorly and the supralevator space superiorly; it is separated from the ischiorectal fossa by the external anal sphincter and the puborectalis muscle, and from the deep postanal space by the upper part of the external anal sphincter and the proximal part of the puborectalis muscle. The anal glands are spread along the walls of the anal canal at the level of the dentate line, published online January 10, 2021
DOI https://doi.org/

$10.1055 / \mathrm{s}-0040-1722356$ ISSN 2581-9933. (c) 2021. Indian Society of Gastrointestinal and Abdominal Radiology. This is an open access article published by Thieme under the terms of the Creative Commons Attribution-NonDerivative-NonCommercial-License, permitting copying and reproduction so long as the original work is given appropriate credit. Contents may not be used for commercial purposes, or adapted, remixed, transformed or built upon. (https://creativecommons.org/licenses/by-nc-nd/4.0/).

Thieme Medical and Scientific Publishers Pvt. Ltd. A-12, 2nd Floor, Sector 2, Noida-201301 UP, India 
which separates the squamous epithelium distally from the columnar epithelium proximally, being situated in the submucosal region and within the internal sphincter. ${ }^{1}$

About $90 \%$ of the perianal sepsis is caused by an obstruction of the ducts of anal glands with subsequent secondary bacterial infection. Types of anorectal abscesses according to their common anatomical locations are perianal abscess, ISP abscess, ischiorectal fossa abscess, and supralevator abscess., ${ }^{2,3}$

The main four types of perianal fistula are the intersphincteric fistula, transsphincteric fistula, suprasphincteric fistula, and extrasphincteric fistula with many subtypes. ${ }^{4}$

About $10 \%$ of anorectal abscesses were not cryptoglandular in origin due to other reasons, among them Crohn's disease, trauma, malignancy, HIV infection, and tuberculosis. ${ }^{2-4}$

The imaging modalities used to diagnose perianal sepsis are the ultrasound via endoanal and transperineal routes using two-dimensional (2D) or three-dimensional (3D) image reconstruction, and magnetic resonance imaging (MRI) through various image sequences and multiplanar image analysis. ${ }^{5.6}$

An abscess appears as closed cavity containing proteinaceous fluid inside (Pus); the fistula tract is a tract with external and internal openings, while a sinus tract is a tract with only one opening and blind end. Fistula tract with an abscess formation is a tract with internal and external openings seen communicating with an abscess cavity; also, a sinus tract with an abscess is a blind end tract with only one opening seen communicating with an abscess cavity. Longstanding sinus or fistula tract showed thick echogenic walls in an ultrasound exam with little or no surrounding fat tissue edema and appeared as a tract with low luminal signal intensity in T1- and T2-weighted images in MRI study, while an active tract showed ill-defined hypoechoic walls with marked surrounding fat tissue edema in ultrasound exam and with increased luminal signal intensity using $\mathrm{T} 2$ and fat suppression image sequences in MRI study. 5,6

The treatment of anorectal abscess depends on incision and drainage, while the treatment of simple perianal fistula depends on fistulotomy or fistulectomy.

The ISP had gained great significance in the pathogenesis and management of various types of anorectal abscesses and perianal fistulas.

\section{Objective}

Descriptive study aimed to identify the various patterns of the ISP sepsis and their incidence among our work group and to clarify the significance of ISP in the pathogenesis of anorectal abscesses and various types of fistula tract, to optimize the way of surgical management.

\section{Methodology}

Retrospective descriptive study of 57 patients with an ISP sepsis, in whom the final diagnosis was based on combining the image findings of the ultrasound, the results of digital rectal examination under anesthesia by an experienced colorectal surgeon, and the operative findings.
All were admitted to the radiology department of our institute from March 2018 to October 2019; among them were 7 females and 50 males, and their age ranged from 20 to 48 years, with the mean age being 37 years.

All were referred from the department of colorectal surgery where they were evaluated by obtaining a full medical history and clinical examination.

Transperineal ultrasound was aided with 3D capability, and for female patients an additional transvaginal ultrasound was performed by an expert radiologist in transperineal and endovaginal ultrasound, using linear $(5-12 \mathrm{MHz})$ and transvaginal (4-9 MHz) multifrequency probes with SonoAce X8 Ultrasound Machine (Medison, Korea).

A 2D transperineal ultrasound was done with the patient in left lateral decubitus position; the knee and hip joints were flexed; the anal canal was examined in the sagittal planes through obtaining midline, left paramedian, and right paramedian sagittal images, and obtaining axial planes at low, mid, and high anal levels, followed by 3D reconstruction of the anal canal with multiplanar image reconstruction and volume rendering. Female patients were further subjected to transvaginal ultrasound through obtaining multiple axial images of the anal canal from low to high anal levels.

The anal canal wall was formed of three layers in an ultrasound exam: the inner echogenic mucosa, the middle hypoechoic internal anal sphincter, and the outer moderate echogenic external anal sphincter ( - Fig. 1 ). The abscess was identified as localized thick wall encysted turbid content fluid collection. Fistula tract was identified as hypoechoic cord-like structure that communicates between an external opening around the anal verge and an internal opening situated in the submucosa of the anal canal wall. The sinus tract was identified as hypoechoic tract with only an internal opening and no external opening. The extrasphincteric course and the relations of abscesses, sinuses, and fistula tracts to the anal canal sphincters were evaluated and the ultrasound images were recorded.

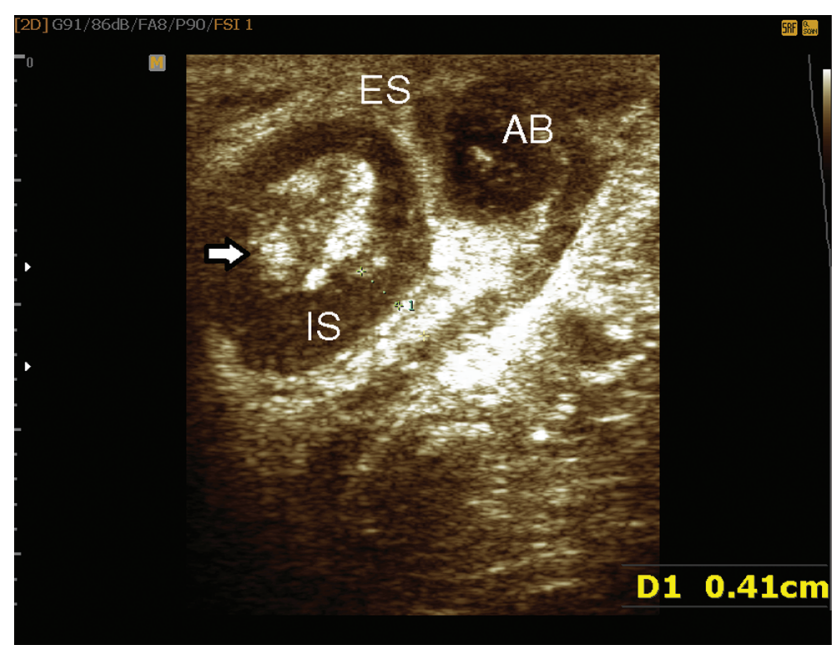

Fig. 1 Normal anatomy of anal canal axial image showing the anal mucosa (arrow), internal sphincter (IS), and the external sphincter (ES) with companion small intersphincteric plane abscess (AB). 


\section{Results}

Overall, 57 patients with an ISP sepsis were included in the study: among them were 50 males (88\%) and 7 females (12\%), with male to female ratio being $7: 1$; also among them, 13 patients (23\%) were detected with an ISP abscess (-Fig. 2), 5 patients (9\%) with free sepsis within the ISP along the anterior, posterior, or the lateral sides of the anal canal wall, 32 patients (56\%) with an intersphincteric fistula tract ( $\sim$ Fig. 3A, B), and 7 patients (12\%) with sinus tracts (-Fig. 4).

Regarding the site of sepsis: Thirty-five patients (61.5\%) were detected with posterior ISP sepsis, 17 patients (30\%) with an anterior sepsis, and 5 patients (8.5\%) with lateral sepsis.

Regarding the longitudinal extension of fistula/sinus tracts: Among 39 patients with fistula and sinuses, the tract was seen extending to the upper $1 / 3$ of the ISP in 12 patients (30.5\%), to the mid $1 / 3$ of the ISP in 21 patients (54\%), and to the lower $1 / 3$ in 6 patients (15.5\%) using an imaginary line, which divides the length of anal canal wall into three divisions.

Regarding fistula/sinus with abscess formation: An intersphincteric fistula with an abscess formation was detected in one patient (2.5\%), an intersphincteric sinus with an abscess formation in one patient (2.5\%), and transsphincteric sinus with the ISP abscess formation was detected in two patients ( $5 \%$ ).

Regarding the shape and site of the abscesses: Among 13 patients with ISP abscesses, 4 patients showed posterior horseshoe intersphincteric abscesses (-Fig. 5), 2 patients had large abscesses extending into the supralevator space with an associated distension of the ISP below the site of the abscesses, and 7 patients showed localized abscess within the ISP.

Regarding complex fistulas: Among the patients with ISP fistula extended to the upper $1 / 3$ of ISP, two patients showed straight nonbranching transsphincteric

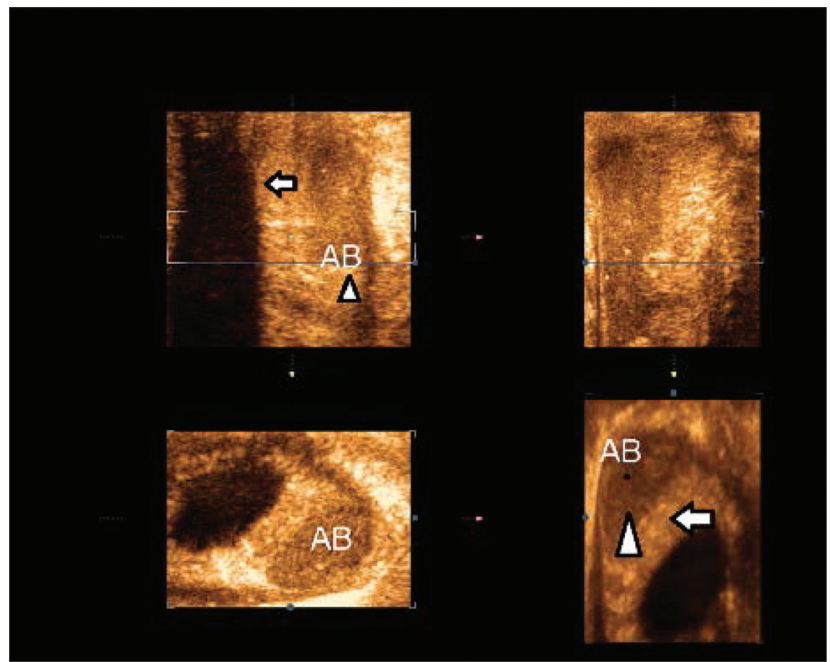

Fig. 2 Transperineal ultrasound three-dimensional multiplanar image analysis with volume rendering of an intersphincteric plane abscess (arrowhead, AB) and the internal anal sphincter (arrow). components seen reaching an external opening away from the anal verge and one patient was detected with posterior ischiorectal fossa horseshoe component ( - Fig. 6) reaching two bilateral external openings located posterior to the anal verge.
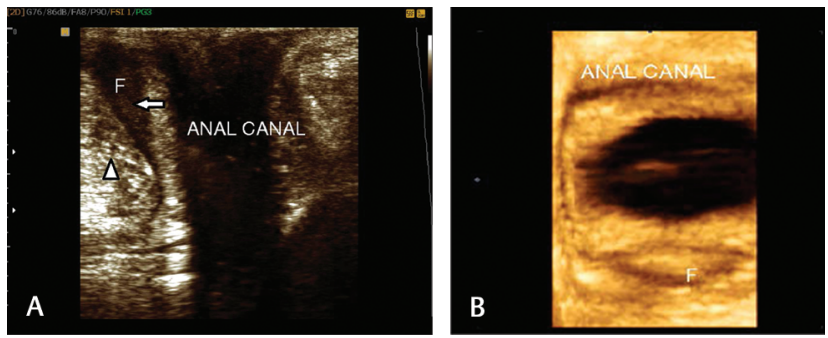

Fig. 3 (A) Two-dimensional transperineal ultrasound of an intersphincteric plane fistula extending to mid $1 / 3$ of anal canal wall (arrow) and the external anal sphincter (arrowhead). (B) Transperineal ultrasound three-dimensional volume rendering of an intersphincteric plane fistula $(F)$.

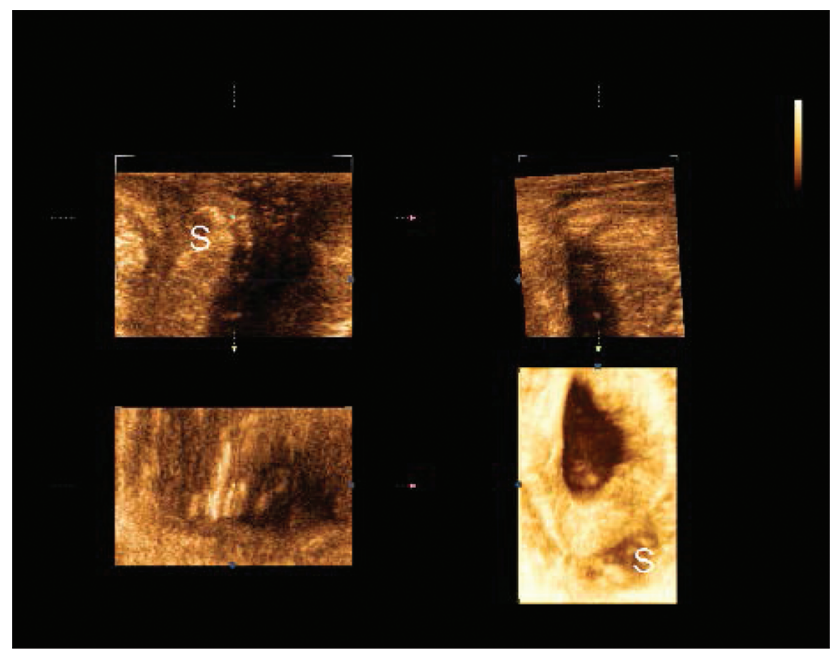

Fig. 4 Transperineal ultrasound three-dimensional multiplanar image analysis with volume rendering of an intersphincteric plane sinus (S).

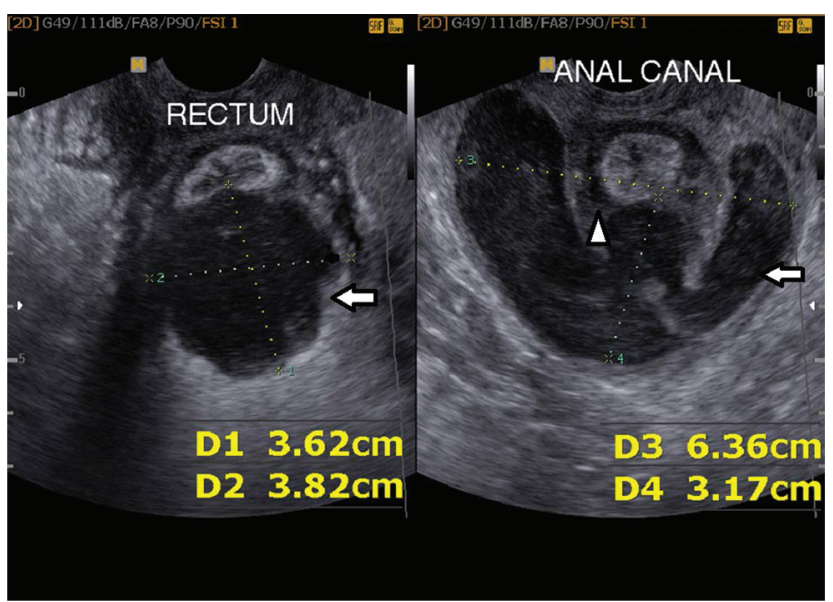

Fig. 5 Transvaginal two-dimensional ultrasound showing large posterior horseshoe intersphincteric plane abscess (arrow) seen locally, destroying the posterior aspect of the internal anal sphincter (arrowhead). 


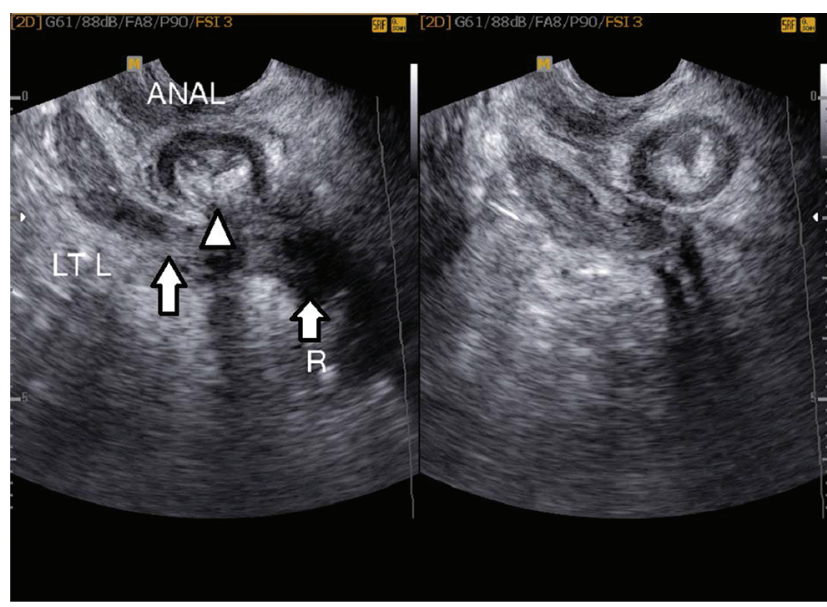

Fig. 6 Transvaginal two-dimensional ultrasound of posterior horseshoe ischiorectal fossa fistula (arrow) seen crossing the external and internal sphincters posteriorly at 6 o'clock (the site of the internal opening, arrowhead).

\section{Discussion}

The current study described many patterns of the ISP sepsis and estimated the incidence of each pattern among our study group. Such patterns could be in the form of fistula tracts, sinus tracts, abscesses, intersphincteric abscess with supralevator extension, sinus with abscess formation, fistula with abscess formation, distension of the ISP with free pus, and other complex forms like horseshoe abscess, abscess with transsphincteric sinus, and ISP fistula/sinus with transsphincteric branches reaching an external opening away from the anal verge.

Such many patterns demonstrated that sepsis can spread from the initial septic focus to all directions of the ISP and from the ISP through the external sphincter to the ischiorectal fossa to form transsphincteric sinuses and fistulas of high and low types; it can also spread from the ISP to the supralevator space and from ISP to the deep postanal space traversing the external sphincter and puborectalis muscle to form horseshoe abscess and subsequent fistula. Such patterns will give an idea about the significance of the ISP in the pathogenesis of cryptoglandular perianal sepsis with subsequent great impact on the way of management.

An obstruction of the anal gland ducts leads to stasis and subsequent secondary bacterial infection..$^{2-4}$ The pathogens start to destroy tissue cells, which attracts macrophages to the site of infection, leading to a fight between the leukocytes and pathogens with resultant liquefactive tissue necrosis and pus formation. ${ }^{7}$ The sepsis extends to the intersphincteric space after localized destruction to the submucosa and the internal sphincter at the site of the infected glands, which leads to the formation of an internal opening mostly at the level of the dentate line (the site of anal glands). The pus that extends to ISP is formed of dead tissue cells, dead and viable bacteria, and dead macrophages. The pus at early stages may be free within the ISP but thereafter becomes walled off with fibrin and viable tissue cells to form a localized abscess. The pus may escape from the intersphincteric space and extend to the perianal space and acquire walls to form perianal abscess or extend to the supralevator space to form supralevator abscess. The infection may spread across the external anal sphincter through localized liquefactive necrosis in the muscle or through the holes of inferior rectal vessels as they penetrate the external sphincter to the ischiorectal fossa to form ischiorectal fossa abscess. The infection may cause an extensive liquefactive necrosis in the ischiorectal fossa and spread to the contralateral side, likely across the deep postanal space, to form ischiorectal fossa horseshoe abscess. ${ }^{8}$ The pus may spread through the ISP in any direction or to its both sides to form an intersphincteric horseshoe abscess.

An external drainage of an abscess, either spontaneous or surgical, may lead to the formation of fistula tract, and insufficient drainage through the internal opening without an external drainage will lead to the formation of sinus tract. So, an external drainage of perianal abscess, which is located inferior and in direct continuity with ISP, may lead to the formation of lower $1 / 3$ ISP fistula. An external drainage of ischiorectal abscess may lead to the formation of transsphincteric fistula, which, according to the site of spread of ISP sepsis across the external sphincter, will be either high or low transsphincteric fistula. An external drainage of supralevator abscess through the ischiorectal fossa will lead to the formation of suprasphincteric fistula tract, which extends to the external opening through the ischiorectal fossa and to the internal opening through the initial site of spread of infection within the ISP. Horseshoe fistula occurs subsequent to external drainage of horseshoe abscess. Extrasphincteric fistula is rarely cryptoglandular in origin unless it shows an extension through the ISP to an internal opening at the dentate line, which can be explained by iatrogenic false passage of a suprasphincteric fistula that creates another external opening in the wall of the rectum. Fistula side branches are either due to false passage or an extension of infection to the surrounding soft tissue planes.

Understanding the pathogenesis will help to reach the optimum way of management. All patterns of cryptoglandular sepsis were assumed to have start point at the internal opening at level of the dentate line, from which it extends initially to the ISP and from there it extends in many directions and through the anal canal walls to the surrounding soft tissue planes and potential spaces. Since the best way to treat cavities that distend with pus (abscess, sinus, and fistula) is adequate drainage, so if the cavity was confined to the ISP, drainage will be through adequate opening of the ISP through the internal opening and the internal sphincter overlying the cavity. If the cavity lies outside the anal canal wall and communicates with the ISP to reach the internal opening, the drainage will be through opening the ISP and laying open the cavity through an external drainage. The role can be applied to different types of cryptoglandular abscesses, fistulas, and sinuses.

Fistulotomy is the best treatment for simple fistula. We lay open the tract from the external to the internal opening and the best treatment for ischiorectal fossa abscess is through incision and drainage with fistulotomy to drain the abscess and associated ISP sepsis. However, fistulotomy cannot be performed for complex fistula that involves a large part of 
the anal sphincters for the fear of anal incontinence. Applying aforementioned role, the treatment of complex fistula can be achieved through laying open the extrasphincteric tract, opening the ISP overlying the intersphincteric fistula component, and widening of the internal opening. A study by Garg concluded that the supralevator fistula almost had intersphincteric component and was managed successfully through laying open the transanal route. ${ }^{9}$ The same principle was applied in the procedure of transanal opening of the intersphincteric space, which was associated with high success rate in managing the complex perianal fistula and in preserving the anal continence, as it preserves the external sphincter. ${ }^{10}$ On the contrary, any procedure that leads to closure of the internal opening like fistula plug, fibrin glue, rectal advancement flap, and ligation of the intersphincteric fistula tract procedures will have a low success rate in managing the complex fistula, since it does not lead to the eradication of the extended sepsis within the ISP that harbors viable pathogens, which by time multiply and lead to fistula recurrence. ${ }^{10-14}$

The deep postanal space lies outside the anal canal wall and is not in direct continuity with the initial sepsis involving the anal glands; it can serve as a route for the extension of posterior ischiorectal fossa sepsis to the contralateral site to form posterior ischiorectal fossa horseshoe abscess or fistula tract. The deep posterior ISP, which is situated in direct continuity with the ISP, plays a more significant role in the pathogenesis of complex fistula tract. ${ }^{15}$

The reported sensitivity of ultrasound in the evaluation of perianal fistula in one study was comparable to that of MRI (87\% for each), with MRI being more specific. ${ }^{16}$ In another study, ultrasound showed high sensitivity in evaluating different types of perianal fistula, with reported sensitivity of $100 \%$ for low transsphincteric fistula, $92 \%$ for high transsphincteric fistula, $96 \%$ for intersphincteric fistula, and of lower sensitivity for extrasphincteric fistula, $78 \%{ }^{17}$

The limitation of ultrasound in the evaluation of perianal sepsis is that it is operator dependent and has lower image resolution than that of MRI.

\section{Conclusion}

Understanding the role of the ISP in the pathogenesis of anorectal abscesses and perianal fistula tracts can help in reaching the optimum way of management.

\section{Ethical Approval}

All procedures followed were in accordance with the ethical standards of the responsible committee on human experimentation (institutional and national) and compliant with the Helsinki Declaration of 1964 and later versions.
Informed consent was obtained from all patients for being included in the study.

\section{Funding}

None.

\section{Conflict of Interest}

None declared.

\section{References}

1 Hassan A, Al Mamun A. Surgical anatomy of anal canal and rectum. In: Chowdri N, Parray F, eds. Benign Anorectal Disorders. New Delhi: Springer; 2016

2 Whiteford MH. Perianal abscess/fistula disease. Clin Colon Rectal Surg 2007;20(2):102-109

3 Rizzo JA, Naig AL, Johnson EK. Anorectal abscess and fistula-in-ano: evidence-based management. Surg Clin North Am 2010;90(1):45-68

4 Parks AG, Gordon PH, Hardcastle JD. A classification of fistula-in-ano. Br J Surg 1976;63(1):1-12

5 Lavazza A, Maconi G. Transperineal ultrasound for assessment of fistulas and abscesses: a pictorial essay. J Ultrasound 2019;22(2):241-249

6 Torkzad MR, Karlbom U. MRI for assessment of anal fistula. Insights Imaging 2010;1(2):62-71

7 Kumar V, Abbas AK, Fausto N. Robbins and Cotran Pathologic Basis of Disease. 7th edition. Philadelphia: Elsevier Saunders; 2005

8 Kurihara $\mathrm{H}$, Kanai T, Ishikawa $\mathrm{T}$, et al. A new concept for the surgical anatomy of posterior deep complex fistulas: the posterior deep space and the septum of the ischiorectal fossa. Dis Colon Rectum 2006;49(10, Suppl):S37-S44

9 Garg P. Understanding and treating supralevator fistula-in-ano: MRI analysis of 51 cases and a review of literature. Dis Colon Rectum 2018;61(5):612-621

10 Garg P. Transanal opening of intersphincteric space (TROPIS) - a new procedure to treat high complex anal fistula. Int J Surg 2017;40:130-134

11 Almeida IS, Wickramasinghe D, Weerakkody P, Samarasekera DN. Treatment of fistula in-ano with fistula plug: experience of a tertiary care centre in South Asia and comparison of results with the West. BMC Res Notes 2018;11(1):513

12 Cirocchi R, Farinella E, La Mura F, et al. Fibrin glue in the treatment of anal fistula: a systematic review. Ann Surg Innov Res 2009;3:12

13 Kim DS. Advancement flap for the treatment of a complex anal fistula. Ann Coloproctol 2014;30(4):161-162

14 Wallin UG, Mellgren AF, Madoff RD, Goldberg SM. Does ligation of the intersphincteric fistula tract raise the bar in fistula surgery? Dis Colon Rectum 2012;55(11):1173-1178

15 Zhang H, Zhou ZY, Hu B, et al. Clinical significance of 2 deep posterior perianal spaces to complex cryptoglandular fistulas. Dis Colon Rectum 2016;59(8):766-774

16 Siddiqui MRS, Ashrafian H, Tozer P, et al. A diagnostic accuracy meta-analysis of endoanal ultrasound and MRI for perianal fistula assessment. Dis Colon Rectum 2012;55(5):576-585

17 Youssef AT. Imaging classification of perianal fistula using the ultrasound. J Gastroenterol Hepatol Res 2015;4(6):1653-1659 